\title{
Revisiting the Relationship among Metrics of Tropical Expansion ${ }^{\mathscr{A}}$
}

\author{
D. W. WAugh,${ }^{\mathrm{a}, \mathrm{b}}$ K. M. Grise, ${ }^{\mathrm{c}}$ W. J. M. Seviour,${ }^{\mathrm{a}}$ S. M. Davis, ${ }^{\mathrm{d}, \mathrm{e}}$ N. Davis, ${ }^{\mathrm{d}, \mathrm{e}}$ O. AdAm, ${ }^{\mathrm{f}}$ \\ S.-W. SON,${ }^{\mathrm{g}}$ I. R. SiMPSON, ${ }^{\mathrm{h}}$ P. W. STATEN, ${ }^{\mathrm{i}}$ A. C. MAYCOCK, ${ }^{\mathrm{j}}$ C. C. UMMENHOFER, ${ }^{\mathrm{k}}$ \\ T. BIRNER, ${ }^{1, \mathrm{n}}$ AND A. MING ${ }^{\mathrm{m}}$ \\ a Department of Earth and Planetary Sciences, Johns Hopkins University, Baltimore, Maryland \\ ${ }^{\mathrm{b}}$ School of Mathematics, University of New South Wales, Sydney, New South Wales, Australia \\ ${ }^{\mathrm{c}}$ Department of Environmental Sciences, University of Virginia, Charlottesville, Virginia \\ ${ }^{\mathrm{d}}$ NOAA/Earth System Research Laboratory, Boulder, Colorado

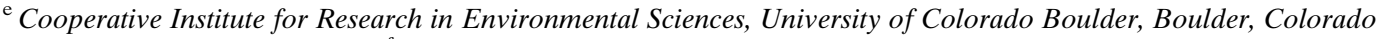 \\ ${ }^{\mathrm{f}}$ Hebrew University of Jerusalem, Jerusalem, Israel \\ ${ }^{\mathrm{g}}$ School of Earth and Environmental Sciences, Seoul National University, Seoul, South Korea \\ ${ }^{\mathrm{h}}$ Climate and Global Dynamics Laboratory, National Center for Atmospheric Research, Boulder, Colorado \\ ${ }^{\mathrm{i}}$ Department of Earth and Atmospheric Sciences, Indiana University Bloomington, Bloomington, Indiana \\ ${ }^{\mathrm{j}}$ School of Earth and Environment, University of Leeds, Leeds, United Kingdom \\ ${ }^{\mathrm{k}}$ Department of Physical Oceanography, Woods Hole Oceanographic Institution, Woods Hole, Massachusetts \\ ${ }^{1}$ Department of Atmospheric Science, Colorado State University, Fort Collins, Colorado \\ ${ }^{\mathrm{m}}$ British Antarctic Survey, Cambridge, United Kingdom
}

(Manuscript received 27 February 2018, in final form 8 June 2018)

\begin{abstract}
There is mounting evidence that the width of the tropics has increased over the last few decades, but there are large differences in reported expansion rates. This is, likely, in part due to the wide variety of metrics that have been used to define the tropical width. Here we perform a systematic investigation into the relationship among nine metrics of the zonal-mean tropical width using preindustrial control and abrupt quadrupling of $\mathrm{CO}_{2}$ simulations from a suite of coupled climate models. It is shown that the latitudes of the edge of the Hadley cell, the midlatitude eddy-driven jet, the edge of the subtropical dry zones, and the Southern Hemisphere subtropical high covary interannually and exhibit similar long-term responses to a quadrupling of $\mathrm{CO}_{2}$. However, metrics based on the outgoing longwave radiation, the position of the subtropical jet, the break in the tropopause, and the Northern Hemisphere subtropical high have very weak covariations with the above metrics and/or respond differently to increases in $\mathrm{CO}_{2}$ and thus are not good indicators of the expansion of the Hadley cell or subtropical dry zone. The differing variability and responses to increases in $\mathrm{CO}_{2}$ among metrics highlights that care is needed when choosing metrics for studies of the width of the tropics and that it is important to make sure the metric used is appropriate for the specific phenomena and impacts being examined.
\end{abstract}

\section{Introduction}

There is mounting evidence that the width of the tropics has expanded over the last few decades and will continue to expand in the future [see, e.g., reviews by

Supplemental information related to this paper is available at the Journals Online website: https://doi.org/10.1175/JCLI-D-180108.s1.

\footnotetext{
${ }^{\mathrm{n}}$ Current affiliation: Meteorological Institute, Ludwig-MaximiliansUniversity, Munich, Germany.
}

Corresponding author: Darryn Waugh, waugh@jhu.edu
Seidel et al. (2008), Birner et al. (2014), and Lucas et al. (2014)]. This expansion could potentially result in shifts in precipitation patterns and ecosystems, with negative consequences on water resources, agriculture, and fisheries (Heffernan 2016). However, there are large differences in reported historical expansion rates (e.g., Davis and Rosenlof 2012), with values ranging from a few tenths to several degrees latitude per decade, and there is currently no consensus on the cause of the expansion. There are several possible reasons for these differences: the observations, reanalyses, and models used differ between studies, as do the time periods and seasons considered, and a variety of metrics have been used to quantify changes in tropical width. 
The possibility that the use of different metrics has contributed to the wide range of reported expansion rates has led to several recent studies examining the covariability of different metrics of the width of the tropics (e.g., Solomon et al. 2016; Davis and Birner 2017). Solomon et al. (2016, hereinafter S16) examined the covariability of seven different metrics of the Southern Hemisphere (SH) tropical edge in a suite of simulations from a single climate model and meteorological reanalyses, and presented evidence that metrics based on the mean meridional streamfunction, lowertropospheric winds, and precipitation minus evaporation are generally correlated with each other on interannual and decadal time scales, but metrics based on outgoing longwave radiation, subtropical upper-tropospheric winds, and tropopause pressure are generally uncorrelated with the streamfunction and precipitation minus evaporation metrics. Davis and Birner (2017, hereinafter DB17) examined covariability of a similar (but not identical) set of tropical width metrics, for both the Northern and Southern Hemispheres, using historical simulations of the twentieth century from phase 5 of the Coupled Model Intercomparison Project (CMIP5; Taylor et al.2012), as well as reanalyses. They also found that $\mathrm{SH}$ metrics derived from the mean meridional streamfunction and surface or lower-tropospheric winds are correlated with each other, and that there was little correlation between these metrics and metrics based on subtropical upper-tropospheric winds or tropopause pressure. DB17 proposed that metrics based on surface or lower-tropospheric winds or the mean meridional streamfunction are related as they all measure different aspects of the total-column eddy momentum driving. On the other hand, metrics based on the subtropical uppertropospheric winds and tropopause pressure are related to the meridional temperature gradient, which is in part related to the stratosphere-troposphere temperature contrast.

While there is much agreement between S16 and DB17 and some general conclusions on covariability of different metrics can be drawn from these studies, there are several inconsistencies. For example, the correlations of SH metrics from models are weaker in DB17 than in S16, and, in contrast to S16, DB17 report a very weak relationship between the precipitation minus evaporation-based metric and other metrics. There is also an inconsistency between S16 and the recent study of Mantsis et al. (2017): Mantsis et al. (2017) reported strong covariability between metrics based on the mean meridional streamfunction and outgoing longwave radiation, contradicting the weak correlations between these metrics reported in S16. It is also notable that the correlations among the metrics examined by DB17 are much weaker in the Northern Hemisphere (NH) than in the SH. In particular, the correlations among metrics based on the mean meridional streamfunction and lower-tropospheric winds are weak or nonexistent in the $\mathrm{NH}$, but are significantly correlated with one another in the SH. The causes of the above inconsistencies or hemispheric differences are as yet unknown, and could be due to a variety of factors including differences in the datasets or model simulations examined, the methods used to calculate the metrics, and the measures of covariability used in the different studies.

Here we revisit the issue of relationships among different metrics of tropical width to identify the robust aspects of the above results and to resolve the differences between them. We also aim to provide guidance on which metrics are appropriate for measuring different aspects of tropical expansion. To do this we examine the covariability of a range of metrics in a large number (23) of CMIP5 models, for different seasons, for the $\mathrm{SH}$ and $\mathrm{NH}$, and in both long preindustrial control (PI) simulations and simulations with an abrupt quadrupling of $\mathrm{CO}_{2}\left(4 \times \mathrm{CO}_{2}\right.$ simulations). By considering 23 models, we can test the robustness of the relationships, while considering seasonal and annual mean fields in both hemispheres, we can examine whether hemispheric or seasonal differences are the cause of any of the above discrepancies. Finally, by considering PI and $4 \times \mathrm{CO}_{2}$ simulations, we can test whether metrics covary for both unforced interannual variability and forced trends.

The models used and metrics calculated are described in the next section. In section 3 we examine the covariability of the metrics on interannual time scales, while in section 4 we examine the spatial variations in the fields used to define the metrics. In section 5 we compare the responses of the metrics to a quadrupling of $\mathrm{CO}_{2}$. Concluding remarks are provided in section 6 .

\section{Data and methods}

\section{a. Models}

Here we use monthly mean output from 23 climate models that participated in CMIP5 (Taylor et al. 2012), provided courtesy of the Program for Climate Model Diagnosis and Intercomparison at Lawrence Livermore National Laboratory. For each model, we analyze the first ensemble member ("r1i1p1") from the PI and $4 \times \mathrm{CO}_{2}$ simulations. The models used are listed in Table 1 . They are the same as in Grise and Polvani $(2014,2016)$, and for each model the PI simulation provides at least 200 years from which to diagnose unforced interannual variability. 
TABLE 1. CMIP5 models and respective length of PI simulation used in analysis. (Expansions of acronyms are available online at http://www.ametsoc.org/PubsAcronymList.)

\begin{tabular}{clc}
\hline \hline Number & \multicolumn{1}{c}{ Name } & PI length $(\mathrm{yr})$ \\
\hline 1 & ACCESS1.0 & 250 \\
2 & BCC_CSM1.1 & 500 \\
3 & BCC_CSM1.1-M & 400 \\
4 & CanESM2 & 996 \\
5 & CCSM4 & 501 \\
6 & CNRM-CM5 & 850 \\
7 & CSIRO Mk3.6.0 & 500 \\
8 & FGOALS-s2 & 501 \\
9 & GFDL-CM3 & 500 \\
10 & GFDL-ESM2G & 500 \\
11 & GFDL-ESM2M & 500 \\
12 & GISS-E2-H & 540 \\
13 & GISS-E2-R & 300 \\
14 & HadGEM2-ES & 239 \\
15 & INM-CM4 & 500 \\
16 & IPSL-CM5A-LR & 1000 \\
17 & IPSL-CM5B-LR & 300 \\
18 & MIROC5 & 200 \\
19 & MIROC-ESM & 531 \\
20 & MPI-ESM-LR & 1000 \\
21 & MPI-ESM-P & 1156 \\
22 & MRI-CGCM3 & 500 \\
23 & NorESM1-M & 501 \\
\hline
\end{tabular}

\section{b. Metrics}

We consider the nine metrics of zonal-mean tropical width listed in Table 2 and shown schematically in Fig. 1. Seven of the metrics were also considered in S16 or DB17, and the two additional metrics have been used in other studies: SLP (Hu et al. 2011; Choi et al. 2014) and $\Delta$ OLR (Davis and Rosenlof 2012; Mantsis et al. 2017). These metrics measure different aspects of the climate system, including the position of the edge of the Hadley cell (PSI metric), the midlatitude eddy-driven jet (EDJ), the transition from surface easterly to westerly winds (USF), the poleward edge of the subtropical dry zone (P-E), the ridge of high sea level pressure in the subtropics (SLP), the subtropical jet (STJ), the subtropical break in the tropopause (TPB), and the width of the region of high outgoing longwave radiation (OLR).

There exist different methods for calculating the metrics based on each climate aspect, but here we consider only one method for each field (see the appendix). The exception is the outgoing longwave radiation field for which we consider two metrics, OLR (as in S16) and $\Delta$ OLR (as in Mantsis et al.2017), to examine if the use of different outgoing longwave radiation metrics is the cause of the conflicting conclusions from these studies (discussed in the introduction). In all cases the metrics are calculated from zonal-mean fields.

\section{c. Analysis}

We use a similar analysis approach to S16: Seasonalmean (DJF, MAM, JJA, SON) and annual-mean zonalmean fields are calculated for each meteorological quantity, and each metric is calculated from these mean fields (e.g., the annual-mean TPB metric is the metric calculated from the annual-mean zonal-mean temperature field). Calculations of some of the metrics have also been performed using monthly mean fields, and comparisons with metrics from seasonal- and annual-mean fields are discussed in section $3 \mathrm{~b}$. The interannual relationship between pairs of metrics is quantified by calculating the Pearson's correlation coefficient $r$.

We also assess the relative year-to-year movement of metrics by calculating the linear regression coefficient $m$ for each metric with PSI, that is, $X=m \mathrm{PSI}+c$, where $X=$ EDJ, SLP, etc., the coefficient $m$ corresponds to the relative change in $X$ for a $1^{\circ}$ change in PSI, and $c$ is a constant. To understand some of the relationships between

TABLE 2. Metrics calculated. Final column lists whether metric was considered in S16 or DB17.

\begin{tabular}{|c|c|c|c|}
\hline Acronym & Climate aspect & Latitude where ... & S16 or DB17 \\
\hline PSI & Hadley cell & Mean meridional streamfunction at $500 \mathrm{hPa}\left(\psi_{500}\right)=0$ & $\mathrm{~S} 16, \mathrm{DB} 17$ \\
\hline EDJ & Eddy-driven jet & $\begin{array}{l}\text { Maximum in } 850 \mathrm{hPa} \text { zonal-mean zonal wind } \\
\quad(\max \mathrm{u} 850) \text { occurs }\end{array}$ & S16, DB17 \\
\hline$P-E$ & Precipitation minus evaporation & Zonal-mean precipitation equals evaporation $(\mathrm{P}-\mathrm{E}=0)$ & S16, DB17 \\
\hline SLP & Subtropical high & Maximum in zonal-mean sea level pressure occurs & - \\
\hline USF & Surface winds & Surface zonal-mean zonal wind is 0 & S16, DB17 \\
\hline STJ & Subtropical jet & $\begin{array}{l}\text { Maximum in } 100-400-\mathrm{hPa} \text { zonal-mean zonal winds with } \\
850-\mathrm{hPa} \text { zonal-mean zonal winds removed } \\
\text { (u100-400 minus u850) }\end{array}$ & S16, DB17 \\
\hline $\mathrm{TPB}$ & Tropopause break & $\begin{array}{l}\text { Maximum in meridional gradient of thermal tropopause } \\
\text { height occurs }\end{array}$ & S16 \\
\hline OLR & Outgoing longwave radiation & Outgoing longwave radiation is $250 \mathrm{~W} \mathrm{~m}^{-2}$ & S16 \\
\hline$\Delta$ OLR & Outgoing longwave radiation & $\begin{array}{l}\text { Outgoing longwave radiation is } 20 \mathrm{~W} \mathrm{~m}^{-2} \text { less than the } \\
\text { subtropical maximum, and poleward of the latitude } \\
\text { of the subtropical maximum }\end{array}$ & - \\
\hline
\end{tabular}




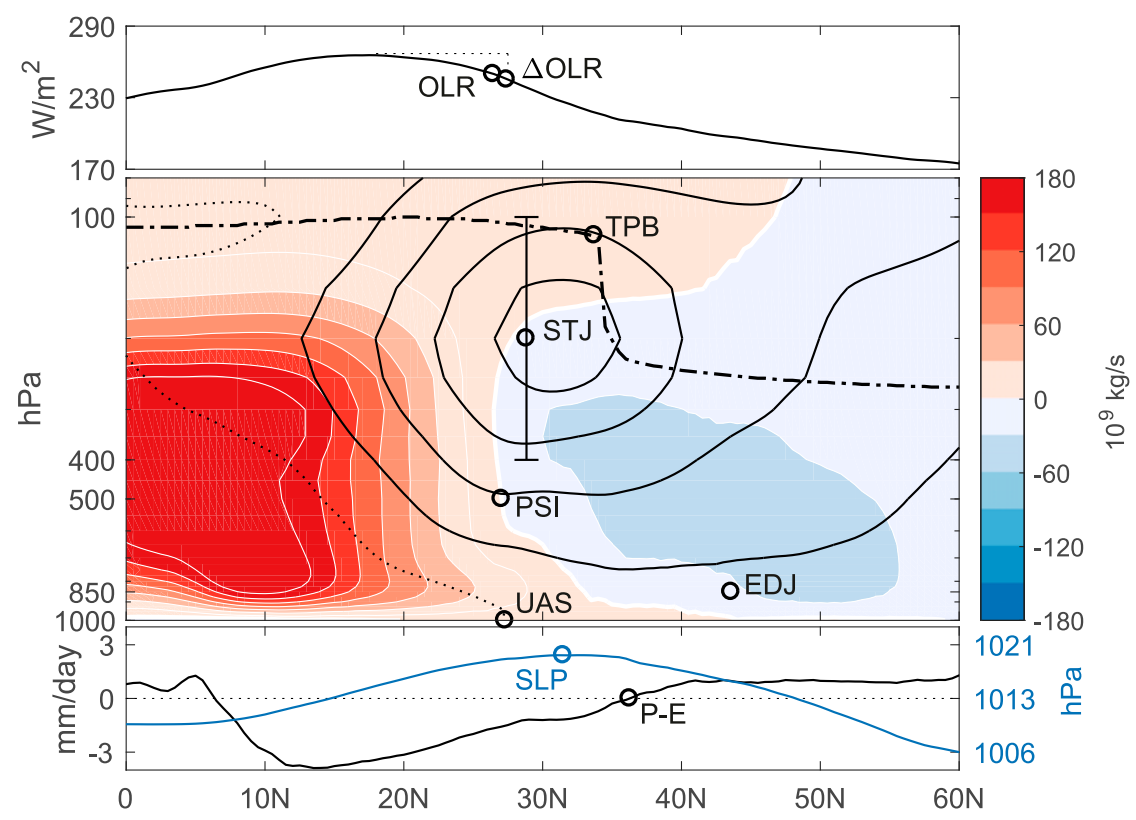

FIG. 1. Schematic illustrating the NH atmospheric circulation and structure, with the location of tropical width metrics listed in Table 2 marked with symbols: (top) outgoing longwave radiation; (middle) zonal winds (black contours; zero: dotted curve), meridional streamfunction (color shading), and tropopause (dot-dashed curve); and (bottom) sea level pressure (blue) and P-E (black). Based on MERRA-2 for February 2002.

metrics we also perform regression analysis for meteorological fields at different locations; that is, $X$ in the above linear regression corresponds to a field (e.g., sea level pressure) at a particular latitude and longitude.

\section{Interannual relationship among tropical width metrics}

\section{a. Climatological locations of metrics}

We first briefly compare the climatological location of the different metrics. As reported previously (e.g., Davis and Rosenlof 2012, S16) there is a range in the mean latitude of different metrics, with most around $30^{\circ}$ in both hemispheres, but some have mean latitudes around $40^{\circ}-45^{\circ}$ (Fig. 2). The latitude of the annual mean for each metric is generally similar (within $5^{\circ}$ ) between hemispheres, and the range and ordering of the latitude of metrics are the same for both hemispheres. Differences in the latitudes of different metrics are expected as the metrics measure different aspects of the climate: for example, EDJ is a measure of the eddy-driven jet, which is expected to be poleward of the Hadley cell edge, whereas $\mathrm{P}-\mathrm{E}$ is near the poleward edge of the subsiding air that is located within the Ferrel cell not at the edge of the Hadley cell where large-scale sinking is maximum (see Fig. 1).

There are also differences between the magnitude and phase of the seasonality of the metrics (different colors of symbols in Fig. 2 represent different seasons). In the $\mathrm{SH}$, metrics are most poleward in summer and equatorward in winter, with the exception of EDJ, which is most poleward in MAM. This is again consistent with previous studies (e.g., S16; Grise and Polvani 2016). In the $\mathrm{NH}$, there is also a tendency for metrics to be most poleward in summer and equatorward in winter, but there is less consistency among metrics.

\section{b. Correlations with PSI}

We now examine the interannual correlation of each of the different metrics with PSI, using fields from the PI simulations. Figures $3 a$ and $3 b$ shows the multimodel mean of the correlation between different metrics and PSI for the $\mathrm{NH}$ and $\mathrm{SH}$, respectively. The standard deviations of the correlations for the model ensemble are also shown in parentheses, and asterisks indicate that at least 20 of the models have a significant correlation (at the $95 \%$ confidence level by a two-sided $t$ test).

The SH correlation coefficients of EDJ, P-E, SLP, and USF with PSI are positive and statistically significant (at the $95 \%$ confidence level) for all models, with the exception of PSI and EDJ in JJA (Fig. 3b). In contrast, there is much less consistency for correlations of TPB or STJ with PSI, with most correlations in individual models being statistically insignificant. The results for correlations of EDJ, P-E, TPB, STJ and OLR with PSI 

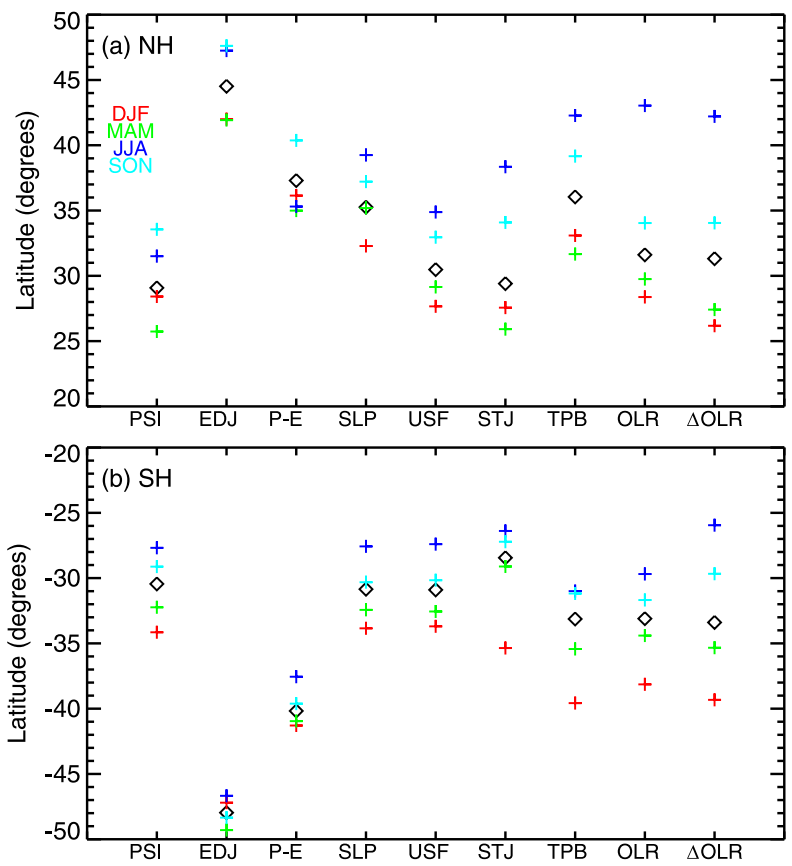

FIG. 2. Multimodel-mean location of each metric, for the annual mean (black diamonds) or for each season, DJF (red plus signs), MAM (green plus signs), JJA (blue plus signs), and SON (cyan plus signs), in the PI simulations.

are generally consistent with S16, who performed the same analysis on a single model (see Fig. 3 of S16). (S16 did not calculate the USF, SLP, or $\triangle$ OLR metrics.)

Figure $3 b$ shows that the use of different OLR-based metrics likely explains the different conclusions between S16 and Mantsis et al. (2017) regarding the relationship of OLR-based metrics and PSI. S16 considered the OLR metric, which is generally only weakly correlated with the PSI metric, whereas Mantsis et al. (2017) examined the annual-mean $\triangle$ OLR, which is correlated with PSI. The reasons for the differences between the two OLR metrics are discussed further below.

We next consider the correlations of metrics with PSI in the NH (Fig. 3a). The results are broadly similar to the SH, with EDJ, USF, and P-E showing higher correlations with PSI than those for STJ, TPB, and OLR. However, there are differences for the PSI and SLP correlations: these two metrics are highly correlated in the $\mathrm{SH}$, but the correlations are much weaker in the $\mathrm{NH}$. This hemispheric difference is related to land-ocean differences between hemispheres (see section $4 \mathrm{~b}$ below). Another hemispheric difference is the seasonality in the EDJ-PSI relationship: there is a large seasonality in the SH (with higher correlation in DJF than JJA) but limited seasonality in the $\mathrm{NH}$.

Consistent with DB17, Fig. 3 shows that there are higher correlations for EDJ, USF, and P-E with PSI than (a) $\mathrm{NH}$

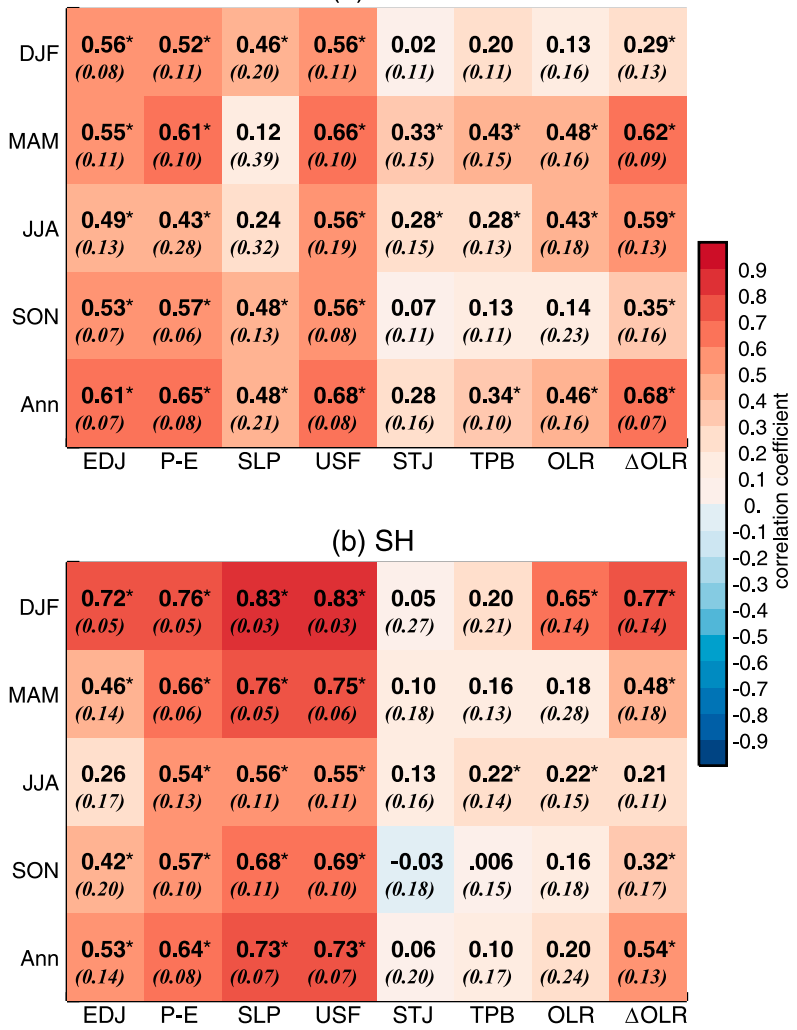

FIG. 3. CMIP5 PI multimodel-mean (and standard deviation in italics) of correlation between different metrics and PSI, for the (a) NH and (b) SH. Results are shown for each season, and for metrics based on annual-mean fields in the PI simulation. An asterisk indicates that at least 20 of the 23 models analyzed have a significant correlation (at the $95 \%$ confidence level).

for STJ and TPB with PSI, in both hemispheres. However, it is notable that the correlations reported here are higher than those for the CMIP5 runs reported in DB17. The mean correlations among PSI, EDJ, and USF are around 0.4 for the SH and $0-0.2$ for the $\mathrm{NH}$ in DB17, compared with values between 0.5 and 0.7 here. There are several differences between the analysis in DB17 and here: DB17 considered CMIP5 historical runs, used a different subset of CMIP5 models, used slightly different definitions or methods of calculation for metrics, and calculated correlations for detrended, deseasonalized monthly mean time series.

To explore the possible causes of the differences from DB17, we repeated the metric calculations performed here using the CMIP5 historical runs (as used in DB17), and these produced very similar correlation coefficients to those from the PI runs (not shown), indicating that differences in the simulations or set of models used do not explain the differences from DB17. We also calculated correlation coefficients from the 23 PI simulations considered here across a range of time scales, including 


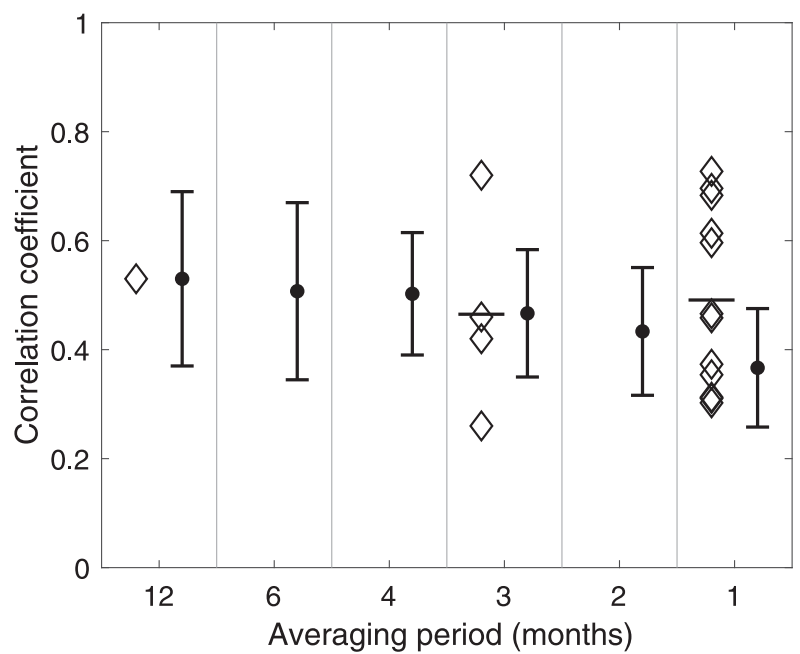

FIG. 4. Multimodel-mean correlation coefficients between PSI and EDJ in the SH for PI simulations, for interannual correlations between the annual-mean, seasonal-mean, or monthly mean metrics (diamonds at 12 months, 3 months, and 1 month), and correlations from deseasonalized monthly mean time series filtered by 1-12 months (black dots for multimodel mean and whiskers for one standard deviation). Horizontal bars show the average of interannual correlations from the seasonal-mean or monthly mean metrics (i.e., average of diamonds). Lowest correlations occur for winter months or season and highest for summer months or season.

the month-to-month correlations used in DB17. The resulting correlation coefficients between PSI and EDJ in the SH are shown in Fig. 4, for (i) multimodel-mean annual, seasonal, and monthly interannual correlations (diamonds, means indicated by horizontal bars) and (ii) multimodel-mean correlations among metrics calculated from 1-, 2-, 3-, 4-, 6-, and 12-month-averaged data (black dots, bars indicate one standard deviation). In this latter calculation the input fields (e.g., zonal wind) are averaged in consecutive, nonoverlapping chunks and the metrics are correlated consecutively. For the 1-6month-averaged data, the time series of the metrics are deseasonalized by subtracting the climatology. In general, as the time scale of the averaging decreases so does the correlation between the metrics, and the month-tomonth correlations yield lower values than the interannual correlations, consistent with the differences between correlations in DB17 and those reported here (and in S16).

A possible contributor to the lower month-to-month correlations could be seasonal differences in the interannual variance of metrics. Interannual correlations relate metrics at the same time of the year and are not affected by seasonality in the variance, but correlating month-to-month time series implicitly fits a single ratio to the time series, resulting in more unexplained variance and a lower correlation when there is large seasonality in the interannual variance of one metric. There is a much larger seasonality in the variance of EDJ than the variance of PSI (e.g., the multimodel-mean interannual variance of EDJ is around 3 times that of PSI for DJF, but 49 times that of PSI for JJA), and consistent with this there is a large decrease in EDJ-PSI correlations from interannual to month-to-month time scales.

While the different types of correlation (month-tomonth or interannual) are a major factor in the differences between DB17 and values reported here, it is not the only factor. Similar calculations to those shown in Fig. 4 for the $\mathrm{NH}$ yield monthly correlations of around 0.4-0.5, which are smaller than values in Fig. 3 but larger than the correlations of less than 0.2 reported in DB17. These lower month-to-month correlations in the $\mathrm{NH}$ in DB17 could be due to differences in how particular metrics are calculated. In particular, the NH streamfunction metric is sensitive to the vertical integration technique used (Adam et al. 2018), and the EDJ metric is sensitive to the vertical level chosen for the zonalmean zonal wind maximum (i.e., $1000 \mathrm{hPa}$ in DB17 vs $850 \mathrm{hPa}$ used here and in S16).

\section{c. Relationship among all metrics}

We now consider the relationships among all pairs of metrics, and not just with PSI. Figure 5 shows these correlations for annual-mean metrics, with the upper left showing the $\mathrm{NH}$ and the lower right the SH. The corresponding plots for each season are shown in Figs. S1-S4 in the online supplemental material.

There are high correlations between all pairs of PSI, SLP, USF, and EDJ in the SH, with USF and SLP nearly perfectly correlated in most models. These four metrics are also generally highly correlated in the $\mathrm{NH}$, but the correlations are typically weaker than in the $\mathrm{SH}$, especially for correlations with SLP. These results generally apply during all four seasons individually (Figs. S1-S4); pairs of lower-atmospheric metrics are generally correlated, with higher correlations in the $\mathrm{SH}$, and with lower correlations with SLP in the NH. The lowest correlations, in both hemispheres, generally occur for MAM and JJA.

The correlations of OLR and $\triangle$ OLR with EDJ, P-E, USF, and SLP are similar to those with PSI, with higher correlations of annual-mean $\triangle \mathrm{OLR}$ with these metrics than for OLR. There are large seasonal variations in the correlations of OLR and $\triangle$ OLR with lower-atmospheric metrics. For example, in the $\mathrm{SH}$ there are high correlations between both OLR and $\triangle$ OLR with other metrics in DJF, but weak (and even negative) correlations of OLR and $\triangle$ OLR with these metrics for other seasons (Fig. 3; Figs. S1-S4).

The STJ and TPB metrics are generally weakly correlated with all other metrics, particularly in the $\mathrm{SH}$, but they do show a high correlation with each other (as also 


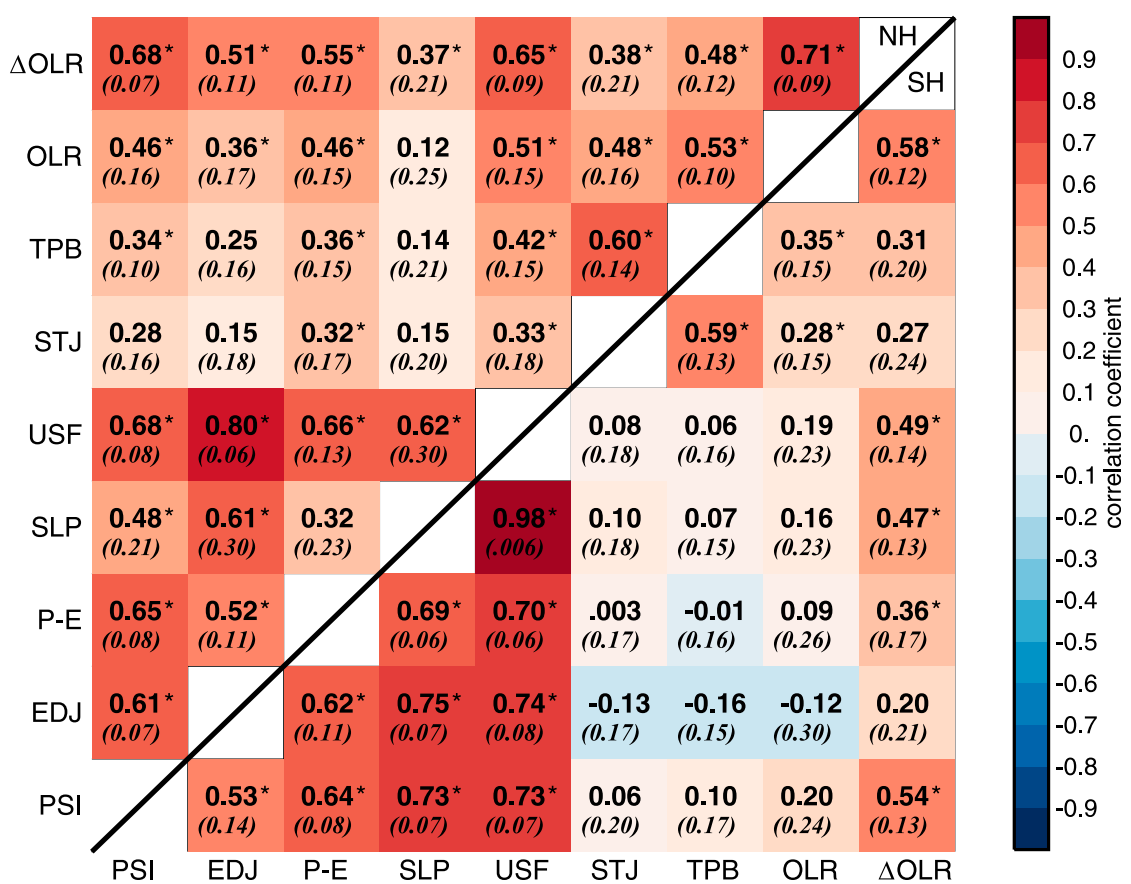

FIG. 5. Multimodel-mean (standard deviation) correlation between pairs of metrics based on annual-mean fields. Upper left is $\mathrm{NH}$ and lower right is SH. An asterisk indicates pairs where more than 20 of the 23 models show statistically significant correlations (at $95 \%$ confidence level).

found by DB17). This also applies for the seasonal-mean metrics (Figs. S1-S4). So the STJ and TPB metrics are likely measuring a similar aspect of the climate system to one another, which is either uncorrelated or only weakly correlated with the variations in the Hadley cell width.

\section{d. Regression coefficients}

The above analysis has identified which tropical width metrics covary interannually, but even if two metrics covary it does not mean that they will shift poleward or equatorward the same amount. The movement of the different metrics in the $4 \times \mathrm{CO}_{2}$ simulations will be examined in section 5 , but first we examine the relative year-to-year movement of metrics in the PI simulations using the regression analysis described in section 2c. Figure 6 shows the values of $m$ (relative change in metrics for a $1^{\circ}$ poleward shift in PSI) for each model, for the (a) $\mathrm{NH}$ and (b) $\mathrm{SH}$ annual-mean metrics, with the color of the symbols showing the correlation coefficient $r$. (For 500-yr simulations, correlations are significant at (a) $\mathrm{NH}$ Annual

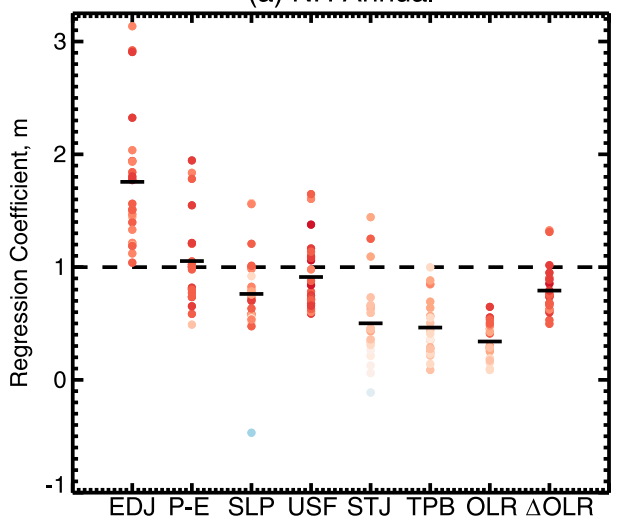

(b) SH Annual

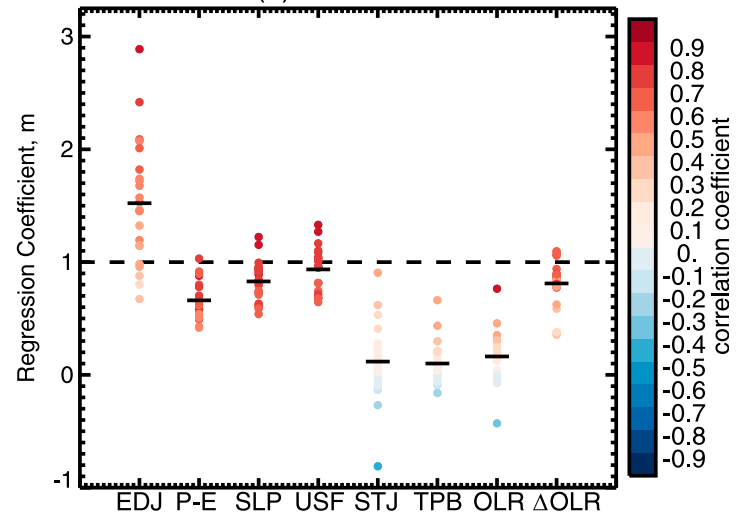

FIG. 6. Regression coefficients of metrics with PSI for annual-mean fields, for the (a) NH and (b) SH from the CMIP5 PI experiment. Symbols show individual models, the horizontal bar shows multimodel mean, and the color of the symbols denotes the correlation coefficient $r$. 
95\% confidence level if $r$ exceeds 0.1 .) The corresponding plots for each season are shown in Fig. S5.

The regression coefficients indicate that EDJ shifts more than PSI, with $m>1$ for nearly all models, for both hemispheres (consistent with earlier studies; e.g., Polvani et al. 2011; Kang and Polvani 2011; Staten and Reichler 2014). There is, however, a large spread among the models with $m$ close to 1 in some models but larger than 2 in others (multimodel-mean $m$ is 1.5 in SH and 1.7 in $\mathrm{NH}$ ); that is, in some models the eddy-driven jet and Hadley cell edge move by the same amount, whereas in others the jet moves twice as far the Hadley cell edge. This spread in $m$ among the models is related to the spread among the models in the distance between the climatological latitudes of PSI and EDJ, with smaller $m$ (smaller relative shift in EDJ compared to PSI) for models with a larger separation between the climatological PSI and EDJ (not shown). The correlation between PSI and EDJ also varies with the climatological distance between PSI and EDJ, with lower correlation for larger climatological difference between PSI and EDJ [consistent with Kidston et al. (2013)]. Indeed, consistent with these relationships, it can be seen, at least for the $\mathrm{SH}$, that models with a smaller $m$ also have a lower correlation (Fig. 6b).

For P-E, SLP, USF, and $\triangle$ OLR the regression coefficient $m$ is closer to 1 , with multimodel-mean $m$ generally less than 1 . Thus we would expect similar variability, and possibly similar shifts in response to forcing, in these metrics to that in PSI. There is still a spread in $m$ among models; in the SH the spread in $m$ for these metrics is less than for EDJ, but in the NH the spread is comparable to that for EDJ. As discussed in Seviour et al. (2018), the spread in $m$ among the models for the P-E-PSI relationship in the $\mathrm{SH}$ is again related to spread in the distance between the climatological latitude of the metrics. The results are generally similar between hemispheres, except for P-E where $m<1$ in the $\mathrm{SH}$ in all but one model (multimodel-mean value around 0.7 ), but $m$ ranges from 0.5 to 2.0 in the $\mathrm{NH}$ (with multimodel-mean $m$ around 1). The cause of this hemispheric difference is unknown and requires further investigation.

For the STJ, TPB, and OLR metrics the regression coefficient $m$ is generally much less than 1 in the models, and can even be negative (multimodel-mean $m$ for the $\mathrm{SH}$ is close to 0 for all 3 metrics). However, the correlation coefficients between these metrics and PSI are smaller than the above metrics and the values of $m$ for these metrics are less robust.

The fact that for several metrics the spread in $m$ among the models is related to the spread in distance between the climatological locations of the metrics suggests that it may be possible to constrain the relative shift of two metrics using their observed climatological relationship. As shown in Seviour et al. (2018), this is not possible for the P-E-PSI relationship because of large uncertainties in the climatological location of $\mathrm{P}-\mathrm{E}=0$ in reanalyses. However, it may be possible for other metrics where there is better agreement in the climatological locations of the metrics among the reanalyses.

\section{Spatial analysis}

To better understand the relationships between PSI and the other tropical width metrics documented above, we perform a regression analysis between PSI and the fields that are used to define the other metrics. The analysis has been performed for both hemispheres and different seasons, with generally similar results. For brevity we focus here on results for SH annual-mean fields, which are summarized in Fig. 7 and Fig. S6.

\section{a. Zonal winds}

We first consider the changes in the $850-\mathrm{hPa}$ zonalmean zonal wind (u850) associated with a meridional shift in PSI. Figure 7a shows the latitudinal distribution of u850 for a $2^{\circ}$ poleward (solid curves) or $2^{\circ}$ equatorward (dashed) shift of PSI from its climatological location. (The corresponding regression coefficients for each individual model, and the mean of the models, are shown in Fig. S6a) Associated with a poleward movement of PSI, there is an increase in winds poleward of, and a decrease equatorward of, the climatological EDJ. This results in a poleward shift of the maximum winds, and hence a poleward shift in the EDJ metric, consistent with the high correlation between PSI and EDJ described above (Figs. 3 and 5). Figure 7a also shows that there is poleward shift of the subtropical zero crossing of u850 associated with a poleward shift of PSI. This is consistent with the high correlation between the USF and PSI metrics (Figs. 3 and 5). The movement in the location of where the $\mathrm{u} 850=0$ is similar to the movement of PSI $\left(4^{\circ}\right)$, but there is a larger movement in the location of maximum u850 (around $6^{\circ}$ ). Again, this is consistent with the previous analysis shown in Fig. 6, where there is a similar shift in USF and PSI $(\sim 1: 1)$ but a larger shift in EDJ than PSI ( 1.5:1).

We next consider the difference between the zonalmean upper-tropospheric $(400-100 \mathrm{hPa})$ and $850-\mathrm{hPa}$ zonal winds $[\Delta u=u(400-100)-\mathrm{u} 850]$, which are used to define the STJ metric. There is near-uniform weakening of $\Delta u$ in the subtropics associated with a poleward shift of PSI (see Fig. S6b), but there is little change in the latitude of the maximum $\Delta u$ that defines STJ (Fig. 7b), consistent with the weak correlation between the PSI 
(a)

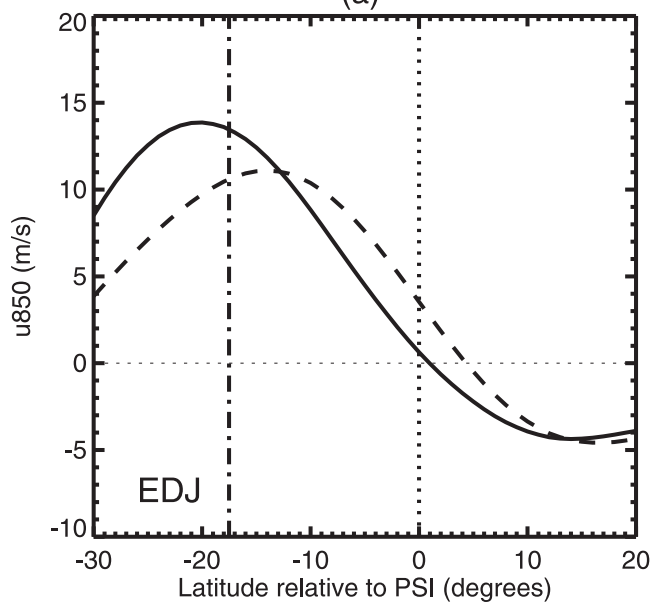

(c)

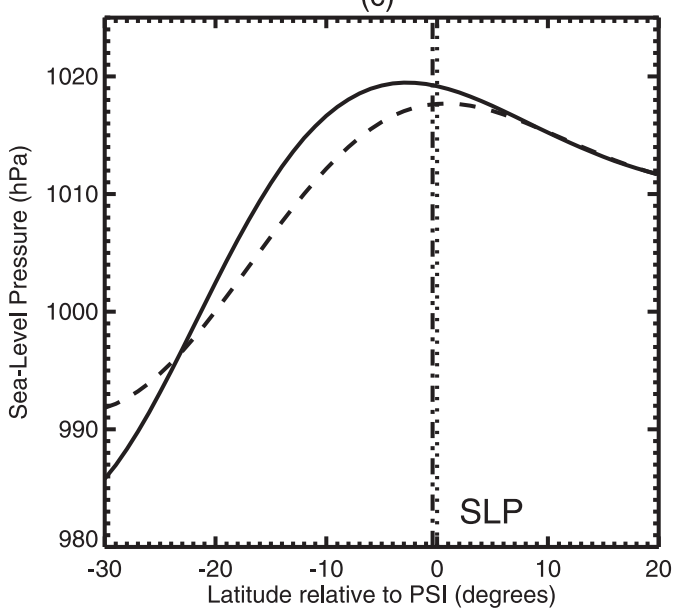

(e)

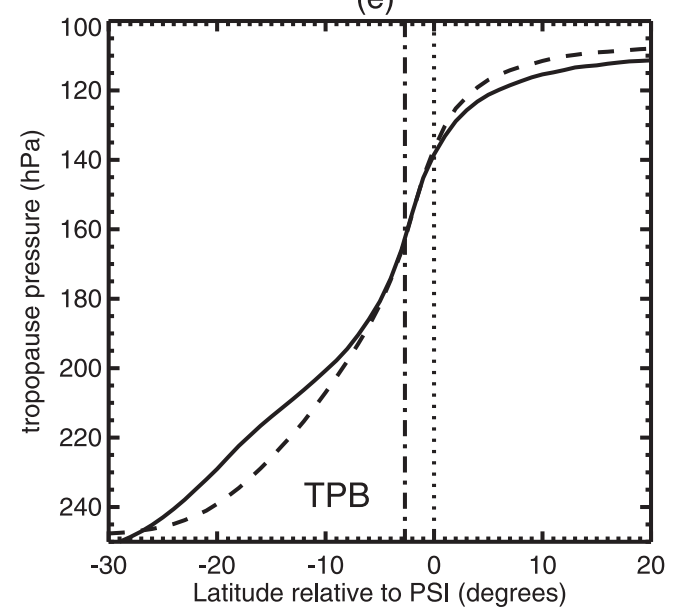

(b)

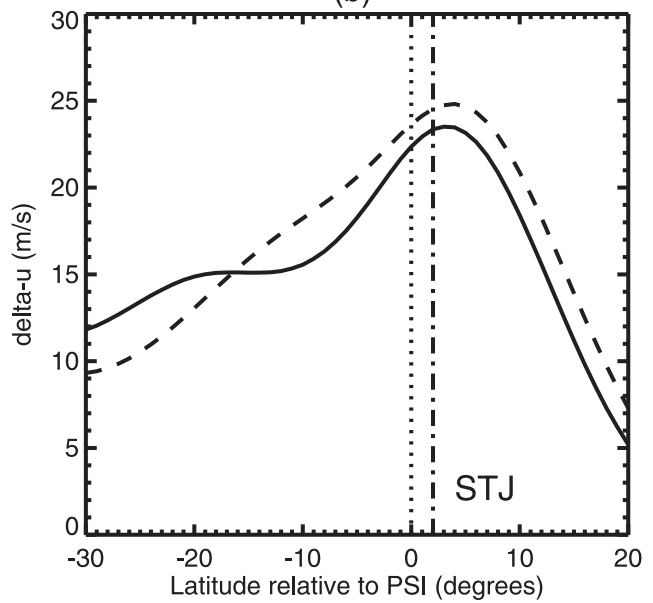

(d)

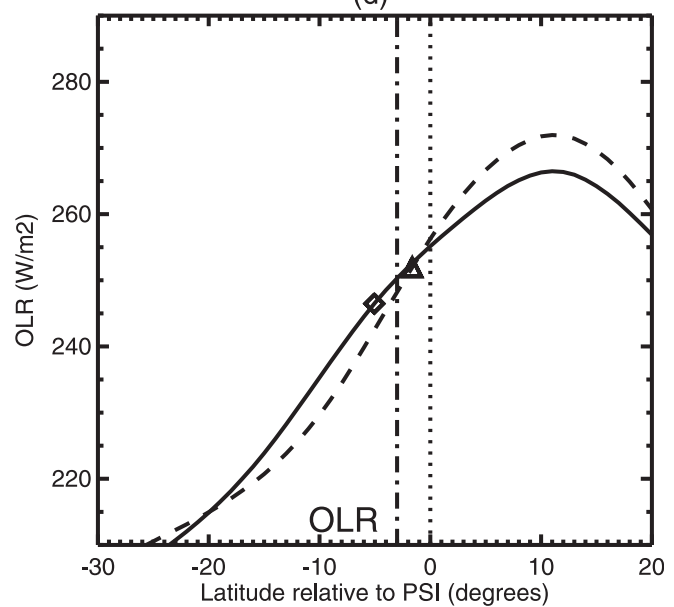

(f)

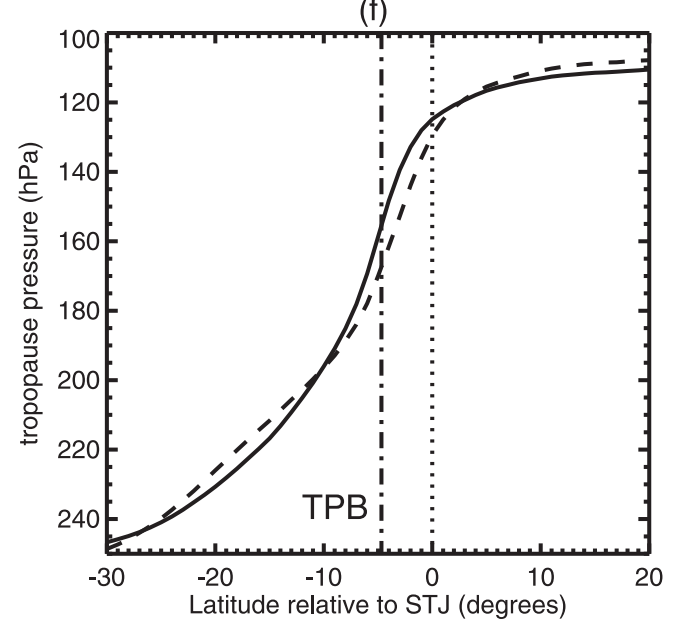

FIG. 7. Latitudinal variation of zonal-mean fields associated with a poleward (solid curve) or equatorward (dashed curve) shift of PSI from its climatological position, for the SH annual mean, for (a) u850, (b) $\Delta u$, (c) sea level pressure, (d) outgoing longwave radiation, and (e) tropopause pressure. (f) As in (e), but for shift of STJ. Regression analysis is performed for each individual model, and plots show multimodel mean. For (a)-(d) the curves correspond to $2^{\circ}$ shifts in PSI from the climatological position, whereas in (e) and (f) they correspond to $10^{\circ}$ shifts. Fields are plotted against the latitude relative to the climatological location of the PSI metric [or STJ for (f)] (vertical dotted line), and the vertical dot-dashed line is the climatological location of the metric. The triangle and diamond in (d) correspond to the mean location of the $\triangle$ OLR metric for an equatorward and poleward shift of PSI, respectively. 
and STJ metrics (Fig. 3). Note that Fig. 7b shows that although the correlation between PSI and STJ is weak, there are still changes in subtropical winds associated with a poleward expansion of the Hadley cell, but these changes are primarily in the magnitude of the winds rather than the latitude of maximum winds.

\section{b. Sea level pressure}

We next consider changes in the zonal-mean sea level pressure field associated with a poleward shift in PSI. There is little change in the sea level pressure equatorward of the climatological SLP (vertical dashed line), which is nearly identical to the climatological PSI (Fig. 7c). However, there is an increase in the sea level pressure poleward of this latitude, and a poleward shift in maximum sea level pressure. This is again consistent with the correlation analysis in section 3 , which showed that PSI and SLP tend to move poleward together in the $\mathrm{SH}$. Figure $7 \mathrm{c}$ also shows an increase in the meridional gradient of SLP between $40^{\circ}$ and $60^{\circ} \mathrm{S}$ (around $10^{\circ}-30^{\circ}$ south of the climatological PSI). This corresponds to a more positive southern annular mode (which can be defined by differences in pressure between $40^{\circ}$ and $60^{\circ} \mathrm{S}$ ) with the poleward expansion of the Hadley cell.

The results shown in Fig. 7c are for SH annual-mean fields. Similar results are found for all seasons in the SH; that is, for each season there is a poleward movement of the latitude of maximum zonal-mean sea level pressure associated with a poleward shift in PSI. There are, however, seasonal differences in the $\mathrm{NH}$ (not shown): In DJF the change in $\mathrm{NH}$ zonal-mean sea level pressure is similar to the $\mathrm{SH}$, with a poleward movement of the latitude of the maximum associated with a poleward shift in PSI, but in JJA there is an insignificant change in the magnitude and location of the maximum of the multimodel-mean zonal-mean sea level pressure. An equivalent analysis of the response in individual models in JJA shows some small changes in sea level pressure with a poleward shift in PSI, but there is a large spread in the location of those changes relative to the SLP maximum among the models. This seasonal difference in the sea level pressure anomalies related to shifts in PSI is consistent with the seasonality in PSI-SLP correlations (Fig. 3), with statistically significant correlations in DJF but not in JJA.

The hemispheric differences in the sea level pressure anomalies associated with shifts in PSI are related to land-ocean differences in the sea level pressure anomalies. Regression analysis shows that there are increases in sea level pressure over oceans associated with a poleward shift in PSI but changes are of inconsistent sign over land (see Fig. S7). This is consistent with Schmidt and Grise (2017), who performed a similar regression analysis using reanalyses and CMIP5 model simulations. In the $\mathrm{SH}$, the oceanic response dominates and there is a large increase in the zonal-mean sea level pressure poleward of the subtropical high, but in the $\mathrm{NH}$ there is partial cancellation of the ocean and land responses and a weaker response in zonal-mean sea level pressure (especially in JJA) (see Fig. S7). In other words, the poleward movement of the Hadley cell is primarily associated with the poleward movement of the subtropical highs over ocean basins.

\section{c. Outgoing longwave radiation}

Next we consider the changes in the zonal-mean outgoing longwave radiation field associated with a poleward shift in PSI. Figure 7d shows that there is an increase in outgoing longwave radiation poleward of the climatological PSI and a decrease equatorward of this latitude associated with a poleward shift of PSI. This also applies for all seasons and for both hemispheres (not shown). These changes in outgoing longwave radiation are consistent with the expected changes in vertical motion associated with a meridional expansion of the Hadley cell. On the equatorward side of the climatological PSI there is anomalous upward motion, whereas there is anomalous downward motion on the poleward side. This results in weaker subsidence and decreased outgoing longwave radiation equatorward of the climatological PSI, and stronger subsidence and increased outgoing longwave radiation poleward of the climatological PSI.

Given this dipole change in zonal-mean outgoing longwave radiation relative to the climatological PSI, the change in the OLR metrics will depend on where the metric lies relative to PSI. The climatological latitude where OLR $=250 \mathrm{~W} \mathrm{~m}^{-2}$ is close to the climatological PSI (which is why it was chosen as a metric) and generally lies between the regions of increasing and decreasing outgoing longwave radiation (Fig. $7 \mathrm{~d}$, vertical dashed line). Thus, there is little change in the latitude where OLR $=250 \mathrm{~W} \mathrm{~m}^{-2}$ and hence weak correlations between the OLR and PSI metrics (Fig. 3).

As the $\triangle$ OLR metric is defined relative to the maximum outgoing longwave radiation, it is influenced by changes in outgoing longwave radiation equatorward of the climatological PSI, as well as possibly changes poleward of the climatological PSI. For the annual-mean fields there is a decrease in peak values of outgoing longwave radiation and the latitudes where outgoing longwave radiation is $20 \mathrm{~W} \mathrm{~m}^{-2}$ less than the new maximum is within the region of increasing outgoing longwave radiation (see symbols in Fig. 7d), resulting in a poleward shift in $\triangle$ OLR. For some seasons (e.g., JJA in the SH, DJF in the NH) the $\triangle$ OLR latitude is equatorward of the 
climatological PSI (and in the region where outgoing longwave radiation decreases with a poleward shift of PSI), and there is a weak or no correlation between $\triangle$ OLR and PSI for these seasons.

Although the latitude where OLR $=250 \mathrm{~W} \mathrm{~m}^{-2}$ is poorly correlated with PSI in the SH annual mean, Fig. $7 d$ suggests that the latitude where OLR = $240 \mathrm{~W} \mathrm{~m}^{-2}$ may be more highly correlated as this latitude lies poleward of the climatological PSI and within the region of increasing outgoing longwave radiation for all seasons. Indeed, there is a higher correlation of OLR $=240 \mathrm{~W} \mathrm{~m}^{-2}$ with PSI than for OLR $=250 \mathrm{~W} \mathrm{~m}^{-2}$ for both hemispheres and all seasons, with the exception of JJA in the $\mathrm{NH}$ where the latitude where OLR = $240 \mathrm{~W} \mathrm{~m}^{-2}$ is at high latitudes (or nonexistent in some models). In the $\mathrm{SH}$ the multimodel-mean correlation of OLR $=240 \mathrm{~W} \mathrm{~m}^{-2}$ with PSI is in the range $0.5-0.6$ for all seasons, whereas in the $\mathrm{NH}$ there is larger variability between seasons (from 0.1 in JJA to 0.6 in MAM). Although the latitude of OLR $=240 \mathrm{~W} \mathrm{~m}^{-2}$ may be more correlated with PSI and other lower-atmosphere metrics than the latitude of OLR $=250 \mathrm{~W} \mathrm{~m}^{-2}$, using this value suffers the same potential issues as OLR = $250 \mathrm{~W} \mathrm{~m}^{-2}$ due to artificial global trends in observational datasets related to changes in satellite instrumentation or sampling as discussed in Davis and Rosenlof (2012).

\section{d. Tropopause pressure}

Finally, we consider the changes in the zonal-mean tropopause pressure. There is much less consistency among the models for the response of tropopause pressure than the fields considered above; for example, the sign of the change in tropopause pressure often varies among the models, and the multimodel change is small (see Fig. S6e). Because of this we show in Fig. 7e the tropopause pressure associated with a $10^{\circ}$ (rather than $2^{\circ}$ ) shift in PSI. Even with this large shift in PSI there is virtually no change in tropopause pressure close to the mean TPB latitude for the multimodel mean (and also in most models), which is consistent with the lack of correlation between TPB and PSI metrics (Fig. 3). As with the OLR and $\Delta u$ fields, although there is no change in tropopause pressure near the latitude of the TPB metric, there are changes in the subtropics and higher latitudes.

\section{e. Subtropical jet and tropopause relation}

The analysis in the previous section showed that the TPB and STJ metrics do not covary with PSI, but that they do covary with each other. To examine this we use the same regression analysis to calculate the change in tropopause pressure associated with a shift in the STJ latitude. There is more consistency among models for the regression of tropopause pressure with a shift in STJ than with a shift in PSI (Fig. S6f), and there is a decrease in tropopause pressure (upward movement of the tropopause) around the TPB latitude with a poleward movement of STJ (see Fig. 7f). The change in tropopause pressure results in a poleward shift in maximum meridional gradients, and, consistent with the correlation analysis (Fig. 5), there is a poleward shift in both TPB and STJ.

\section{Response to $\mathrm{CO}_{2}$ forcing}

Finally, we consider the response in the different metrics in the $4 \times \mathrm{CO}_{2}$ simulations and examine whether the forced response is the same for all metrics. Grise and Polvani (2016) (see also Grise and Polvani 2014) have calculated the response in PSI, P-E, and EDJ in the $4 \times \mathrm{CO}_{2}$ simulations of the same 23 models considered here. We extend this analysis to consider the other metrics of tropical width. We also compare the relative responses of different metrics with the regression analysis from the PI runs. As in the regression analysis, we focus on metrics calculated from annual-mean fields.

\section{a. Southern Hemisphere}

We consider first the SH. Figure 8 shows scatterplots between the response of each metric to $4 \times \mathrm{CO}_{2}$ forcing versus the response of PSI to the same forcing, where the response is defined as the difference between the average of years $50-150$ of the $4 \times \mathrm{CO}_{2}$ simulation from the climatological value for the PI run. As shown in Grise and Polvani (2016), there is a poleward shift in the annual mean PSI, P-E, and EDJ in all models, with a larger shift in EDJ than the other metrics (multimodel-mean shift of EDJ is $\sim 2.75^{\circ}$ compared to $\sim 1.7^{\circ}$ for PSI and P-E). There is also a poleward shift in SLP and USF, with similar multimodel-mean shifts as PSI. The same result is also reported by Son et al. (2018) for the extended representative concentration pathway (RCP) 4.5 simulations.

For all metrics there is a large spread in the magnitude of the shift among the models (e.g., $1^{\circ}-3^{\circ}$ shift in PSI), and models with larger shifts in PSI tend to have larger shifts in EDJ, P-E, SLP, and USF (correlation coefficients between shift in each metric and PSI are listed in Fig. 8). The slope of the linear fit between each metric and PSI also varies among the metrics, ranging from 0.66 for P-E to 1.4 for EDJ (see Fig. 8). These values are close to the multimodel-mean regression coefficient for interannual variations in the PI runs (Fig. 6b), as can be seen by comparing the slopes of the solid and dashed lines (which show linear fit to trends and regression based on interannual variations, respectively). 
(a) PSI and EDJ

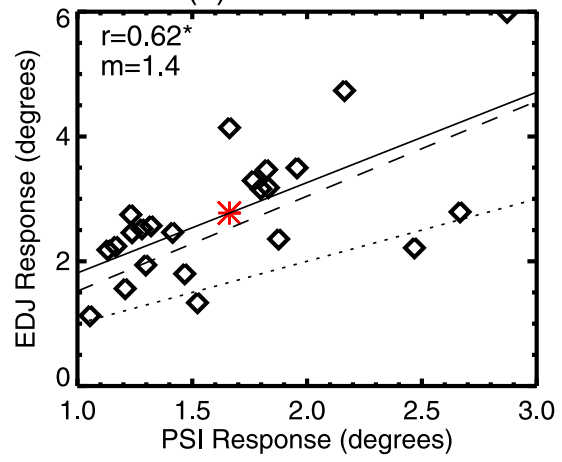

(c) PSI and SLP

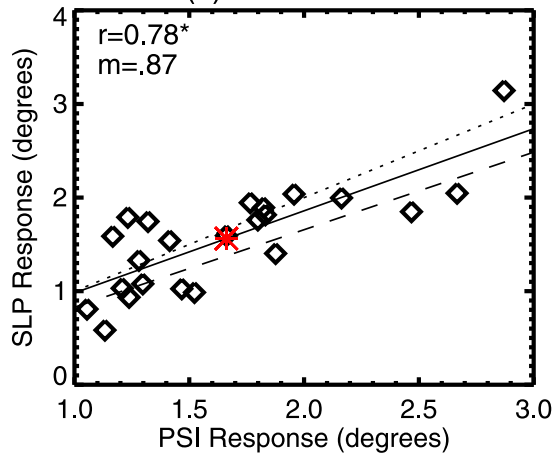

(e) PSI and STJ

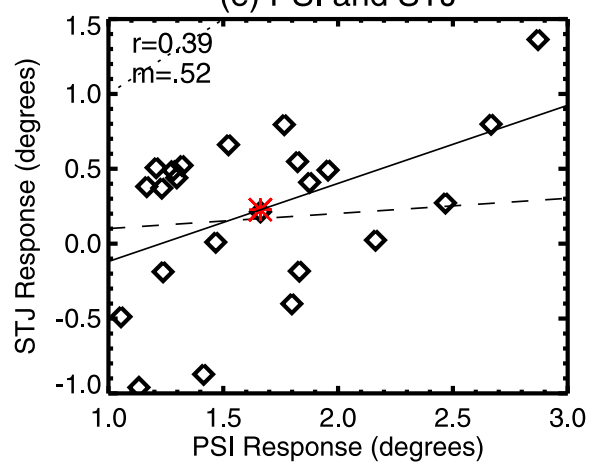

(g) PSI and OLR

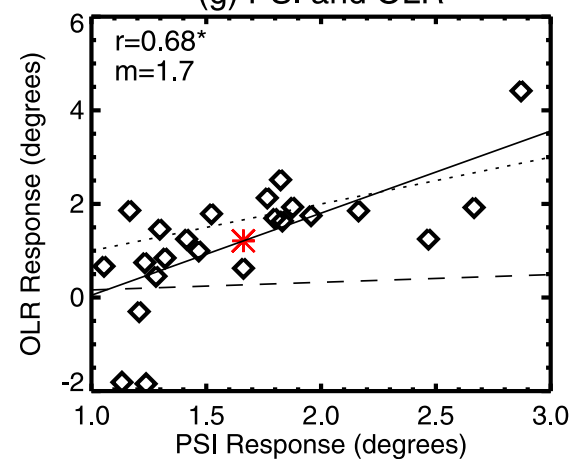

(b) PSI and P-E

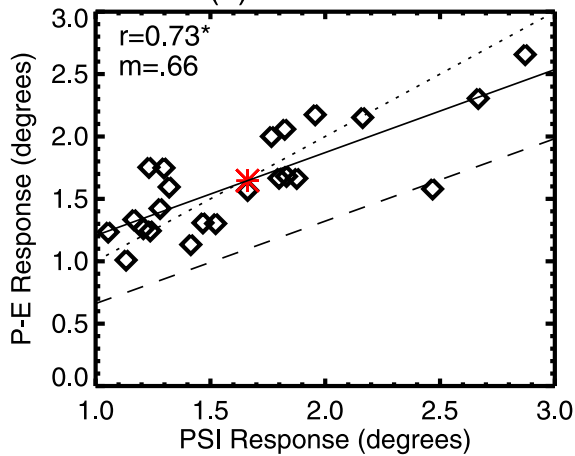

(d) PSI and USF

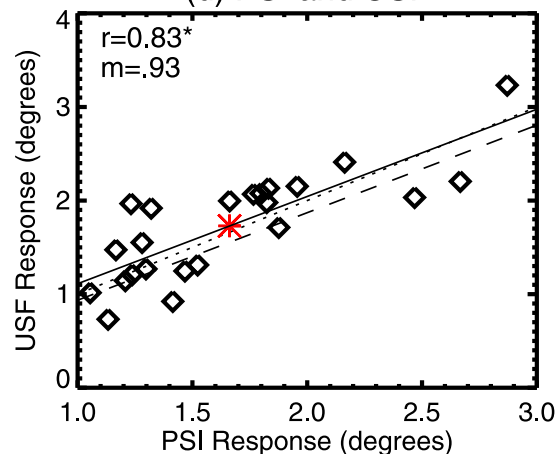

(f) PSI and TPB

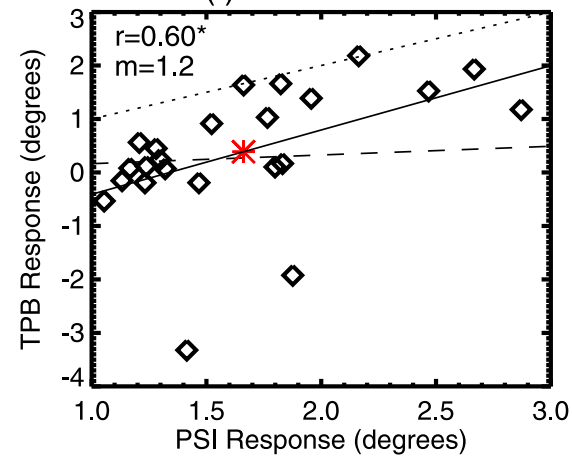

(h) PSI and $\triangle \mathrm{OLR}$

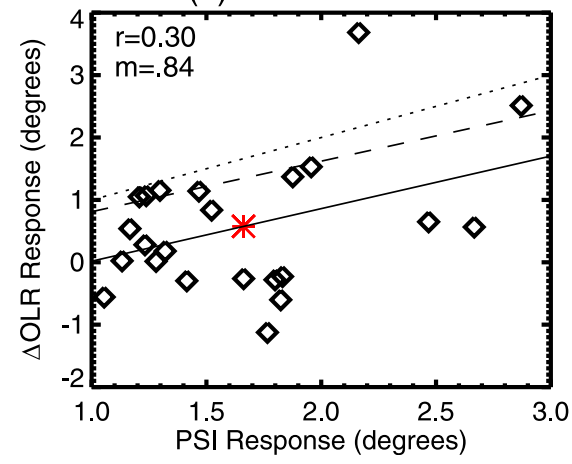

FIG. 8. Relationships between the response of SH annual mean metrics to $4 \times \mathrm{CO}_{2}$ with that of PSI. Each diamond is a different model, and red asterisks are the multimodel means. The solid lines are the linear fits to 23 models, the dashed lines have slope equal to the regression coefficient $m$ from the interannual regression analysis (Fig. 6), and the dotted lines indicate the 1:1 lines. Correlation coefficient $r$ (asterisk indicates significance at 95\% confidence level) and slope $m$ are given in the legend. 
The shift in OLR across the models is also correlated with that in PSI $(r=0.68)$ and the multimodel-mean shift in OLR is comparable with (although slightly smaller than) that for PSI. This is surprising given that there is a very weak correlation between OLR and PSI on interannual time scales in the PI simulations (Fig. 3), and we would not expect the OLR and PSI trends to be highly correlated based on our interannual correlations alone (dashed line shows expected relationship). The cause of this apparent contradiction is that the trends in the OLR metric in the $4 \times \mathrm{CO}_{2}$ run are due to changes in the global-mean OLR rather than the expansion of the Hadley cell. In the $4 \times \mathrm{CO}_{2}$ runs there is an initial decrease in global-mean OLR, but the OLR recovers to PI values over the next few decades because shortwave feedbacks cause an increase in the net absorption of solar radiation (Donohoe et al. 2014). In the majority of the models the global-mean OLR exceeds the PI values by the end of the 150-yr run, resulting in the poleward shift in the OLR metric shown in Fig. 8g. Further, the intermodel spread in OLR response is highly correlated with the climate sensitivity of the models (correlation coefficient $r=0.78$ ), as the climate sensitivity of the models is largely determined by shortwave cloud feedbacks (e.g., Vial et al. 2013). The spread in PSI across the models is also highly correlated $(r=0.81)$ with the climate sensitivity of the models (Grise and Polvani 2014, 2016; Davis et al. 2016), and this results in the correlated response of the OLR and PSI metrics.

The correlation between shifts in $\triangle O L R$ and shifts in PSI (Fig. 8h) is weaker than the correlation found on interannual time scales between $\triangle$ OLR and PSI (Fig. 3). Unlike the shifts in PSI and OLR, the shifts in $\triangle$ OLR are not correlated with the climate sensitivity of the models $(r<0.01)$, as they are only weakly affected by the globalscale changes in OLR discussed above. This insensitivity to changes in global-mean OLR occurs because $\triangle$ OLR is defined relative to maximum OLR (which generally increases in the $4 \times \mathrm{CO}_{2}$ runs). Note that, while the relationship is weaker, the slope of the linear fit between the responses of $\Delta \mathrm{OLR}$ and PSI to $4 \times \mathrm{CO}_{2}$ is consistent with the multimodel-mean regression coefficient for interannual variations in the PI runs (see solid and dashed lines in Fig. 8h).

The shifts in STJ and TPB in response to $4 \times \mathrm{CO}_{2}$ are much smaller than the shift in PSI, with the multimodelmean shift less than $0.5^{\circ}$ and an equatorward shift for several of the models. There is however still a moderate correlation between the shift in STJ or TPB with the shift in PSI across the models (the correlation between TPB and PSI is significant at the $95 \%$ confidence level, but the STJ and PSI correlation is not), which is in contrast to the small correlations found from interannual variability in the PI simulation (Fig. 3). The correlation among models for trends in the $4 \times \mathrm{CO}_{2}$ simulation may again be due to trends in the STJ and TPB metrics due to radiative changes (e.g., Voigt and Shaw 2015) and not necessarily associated with trends in the edge of the Hadley cell.

The above results are consistent with the earlier S16 and DB17 studies that showed consistent forced responses in PSI, EDJ, P-E and USF, but weak (or opposite) responses in STJ, TPB, and OLR.

\section{b. Northern Hemisphere}

The responses of the metrics in the NH (Fig. 9) are somewhat similar to the $\mathrm{SH}$, with generally positive correlations with PSI trends (i.e., models with larger PSI response tend to have a larger response in the other metrics). However, there are some differences. The poleward movement of PSI in the $\mathrm{NH}\left(0.2^{\circ}-1.2^{\circ}\right)$ is much smaller than in the $\mathrm{SH}$. There is also generally a poleward movement of P-E, USF, and EDJ in the NH, but unlike in the $\mathrm{SH}$ for each metric two or three models show an equatorward shift (although they are not the same models for each metric). In the SH there is a strong correlation between the TPB and PSI trends but this is not the case in the $\mathrm{NH}$.

One of the most notable differences between hemispheres is the trends in SLP. Whereas SLP shifts poleward in the $\mathrm{SH}$, there is an equatorward movement of SLP in the NH in most models. So although there are moderate interannual correlations between annualmean SLP and PSI in the NH, these metrics do not respond the same to increases in greenhouse gas forcing, and in fact they can show different signed trends. Analysis of the spatial structure of the multimodel-mean sea level pressure response to $4 \times \mathrm{CO}_{2}$ shows an increase in pressure on the poleward side of the Atlantic subtropical high (consistent with a poleward shift of SLP) but an increase on the equatorward side of the Pacific high [not shown, but spatial pattern is very similar to the response in CMIP5 historical greenhouse-gas-onlyforced simulations shown in Fig. 4 of Schmidt and Grise (2017)]. The above indicates that although PSI and SLP may covary interannually, the SLP metric is probably not a good metric for monitoring forced changes in the Hadley cell width in the NH.

Another difference is the relationship between the PSI and OLR responses. As in the SH, both PSI and OLR in the $\mathrm{NH}$ move poleward with an increase in $\mathrm{CO}_{2}$. However, there is a weaker correlation between the PSI and OLR responses in the NH. This occurs because while there is again a high correlation $(r=0.77)$ between the climate sensitivity and the OLR response in the $\mathrm{NH}$, there is a much weaker correlation $(r=0.38)$ between 
(a) PSI and EDJ

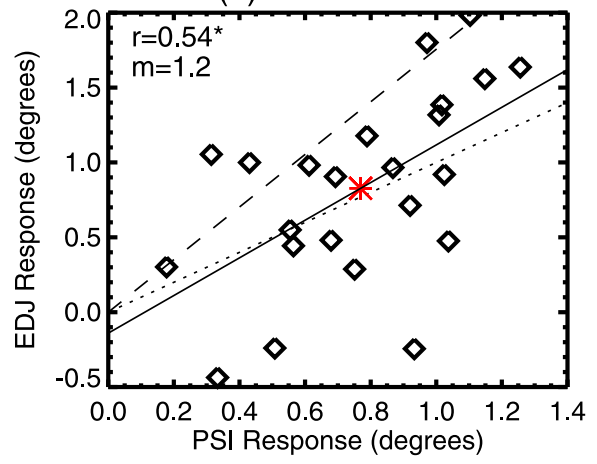

(c) PSI and SLP

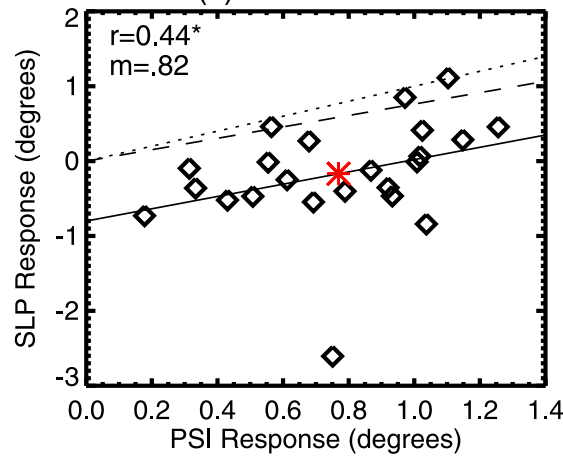

(e) PSI and STJ

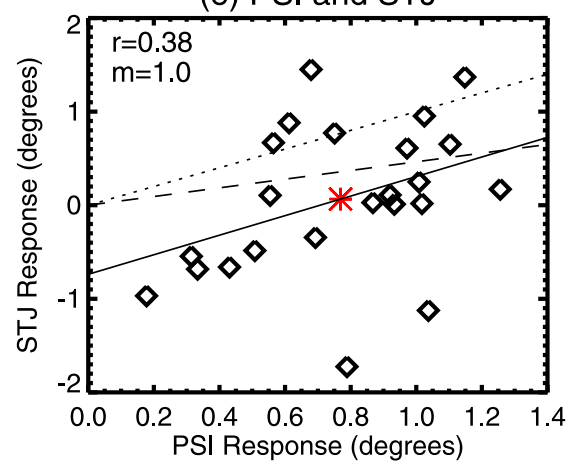

(g) PSI and OLR

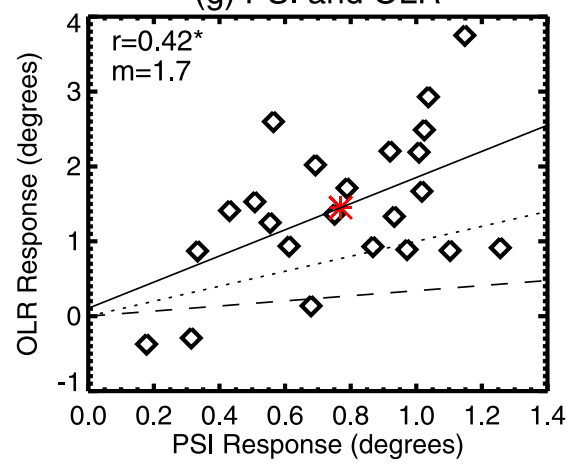

(b) PSI and P-E

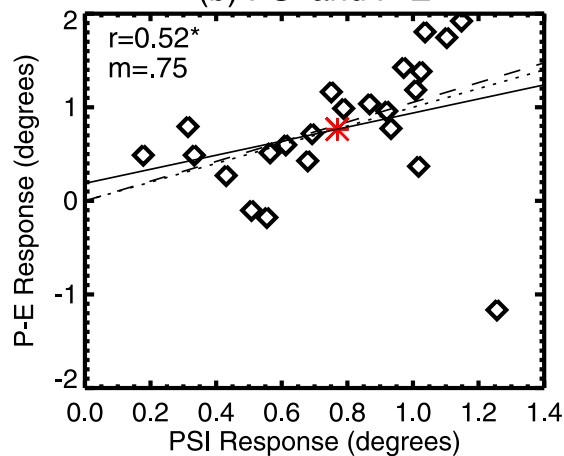

(d) PSI and USF

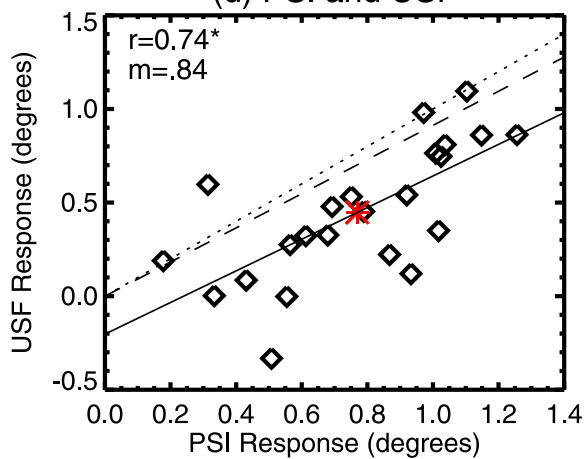

(f) PSI and TPB

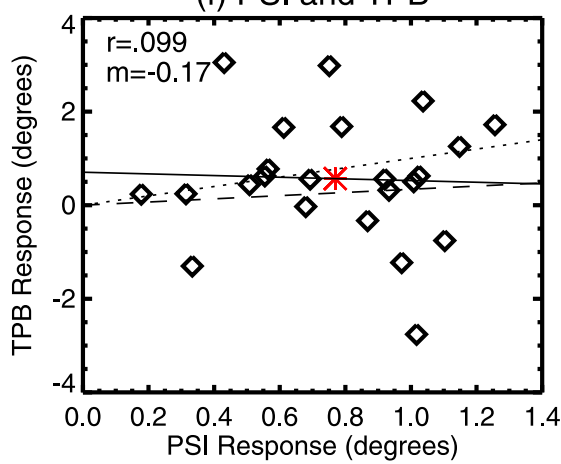

(h) PSI and $\triangle$ OLR

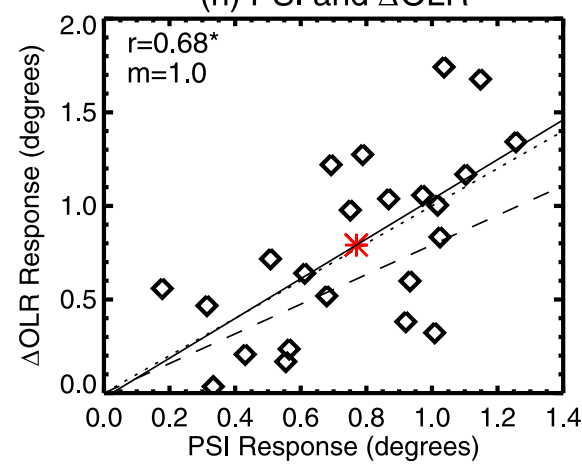

FIG. 9. As in Fig. 8, but for the NH annual mean. 
the climate sensitivity and PSI response in the $\mathrm{NH}$ (Grise and Polvani 2016).

\section{Conclusions}

In this paper, we have provided a detailed analysis of the relationships among nine metrics of the width of tropics used in past studies to quantify the recent expansion of the tropics. Analysis of control and $4 \times \mathrm{CO}_{2}$ simulations from 23 CMIP5 models shows the following:

1) The PSI, USF, P-E, and EDJ metrics generally covary on interannual time scales for both hemispheres and all seasons, and all metrics shift poleward in response to quadrupling of $\mathrm{CO}_{2}$. There is, however, a large spread in the relative year-to-year movement of metrics among the models.

2) The SLP metric covaries with the above four metrics and also shifts poleward in the response to increased $\mathrm{CO}_{2}$ in the $\mathrm{SH}$. However, this is not generally the case in the $\mathrm{NH}$ where interannual correlations of SLP with other metrics can be very low, and SLP moves equatorward in many $4 \times \mathrm{CO}_{2}$ simulations.

3) Whether OLR-based metrics covary with PSI (and the above metrics) depends on the OLR metric used. In general, the latitude of OLR $=250 \mathrm{~W} \mathrm{~m}^{-2}$ does not covary with PSI or other metrics in the $\mathrm{SH}$, but $\Delta \mathrm{OLR}=20 \mathrm{~W} \mathrm{~m}^{-2}$ does for annual mean fields. The response of the two OLR metrics to $4 \times \mathrm{CO}_{2}$ is driven primarily by the increases in the net absorption of solar radiation (due to shortwave feedbacks) and not changes in tropical circulation, and these metrics are not good indicators of greenhouse gas-forced changes of the width of the Hadley cell.

4) The STJ and TPB metrics do not strongly covary with the other metrics considered. However, they are generally correlated with each other, and STJ and TPB may be measuring similar aspects of the circulation, which are uncorrelated with Hadley cell expansion or contraction.

The above results are generally consistent with the conclusions of S16 and DB17, and also explain the discrepancies (highlighted in the introduction) among these and other studies of tropical expansion. In particular, the higher values for correlations among metrics in S16 compared to DB17 are largely due to S16 calculating interannual correlations from annual-mean data and DB17 calculating month-to-month correlations among metrics. Also, the different conclusions in S16 and Mantsis et al. (2017) regarding covariability of PSI and OLR metrics are due to different metrics used in the two studies: $\mathrm{S} 16$ used the latitude where OLR $=250 \mathrm{~W} \mathrm{~m}^{-2}$, which is not well correlated with PSI, whereas Mantsis et al. (2017) used the $\triangle$ OLR metric, which is correlated with PSI (at least for annual-mean fields).

The results presented here, together with those of S16 and DB17, show that the PSI, USF, P-E, EDJ, and SH SLP metrics are closely related to the width of the Hadley cell (or subtropical dry zone; latitude where $\mathrm{P}-\mathrm{E}=0$ ), and use of these metrics will likely give similar measures of tropical expansion. Although EDJ covaries with tropical width on interannual time scales, the magnitude of its forced response can be very different from that of direct measures of tropical width such as PSI. On the other hand, SLP in the NH, OLR-based metrics, STJ, and TPB should not be used as measures of expansion of the Hadley circulation (or dry zones) because they either do not strongly covary with the above metrics or respond differently to increases in greenhouse gases. This is unfortunate as these metrics include those that can be derived from observations rather than meteorological reanalyses (e.g., OLR from satellite data, TPB from radiosondes, and SLP from surface observations).

There are several open questions that remained unanswered by this study. One is this: What is the physical explanation for weak relationship between variations in the STJ and TPB compared with those in PSI and other Hadley cell width metrics? A second, somewhat related, question is this: Do other metrics based on upper-tropospheric winds or tropopause pressure (e.g., Lucas et al. 2014; Amaya et al. 2018) have a stronger correlation to PSI? The analysis in section 4 shows that some aspects of the upper-tropospheric winds and tropopause pressure do vary with PSI, so modified metrics may have a stronger relationship with PSI.

A general, take-home message from this study is that care is needed when choosing metrics for studies of the width of the tropics, and it is important to make sure the metric used is appropriate for the specific phenomena and impacts being examined. Similar care is needed if introducing a new metric - it is important to show that the metric varies in a similar manner to the property of interest (e.g., Hadley cell width) and it is not enough to have a similar climatological-mean location.

Acknowledgments. We thank Karen Rosenlof for helpful comments and discussions on earlier versions of the manuscript, and Damianos Mantsis for initial discussions regarding OLR metrics. This paper arises from discussions within the International Space Science Institute (ISSI) Tropical Width Diagnostics Intercomparison Project and the U.S. Climate Variability, Predictability and Change (U.S. CLIVAR) Working Group on the Changing Width of the Tropical Belt. The authors 
thank the entire members of these groups, the ISSI and U.S. CLIVAR offices, and sponsoring agencies (ESA, Swiss Confederation, Swiss Academy of Sciences, University of Bern, NASA, NOAA, NSF, and DOE). All model data used in this paper are freely available through the Earth System Grid Federation (https:// pcmdi9.1lnl.gov/projects/esgf-lln1/). We acknowledge the World Climate Research Programme's Working Group on Coupled Modelling, which is responsible for CMIP, and we thank the climate modeling groups for producing and making available their model output. For CMIP, the U.S. Department of Energy's Program for Climate Model Diagnosis and Intercomparison provides coordinating support and led development of software infrastructure in partnership with the Global Organization for Earth System Science Portals. DW acknowledges support from NSF AGS-1403676.

\section{APPENDIX}

\section{Calculation of Metrics}

All metrics were calculated using the Tropical-width Diagnostics (TropD) code (Adam et al. 2018). For all metrics the basic fields used for the calculations are zonally averaged and also averaged in time before calculations of the metrics (i.e., the zonal-mean meridional velocity is averaged over season or year before calculation of the mean meridional streamfunction, and the zonal-mean temperature is averaged in time before calculation of the thermal tropopause).

PSI is the zero-crossing latitude of the mean meridional streamfunction at the $500-\mathrm{hPa}$ level, poleward of the tropical streamfunction extremum in either hemisphere. If an additional zero-crossing latitude that meets the same criteria is found within $20^{\circ}$ latitude poleward of the initial zero-crossing latitude, the metric is deemed poorly defined for that month and is set to a missing value. The zonal-mean meridional mass streamfunction $\psi$ is calculated from the zonal mean of the meridional wind (integrating from the uppermost pressure level down to the surface level). The metric is calculated using the "Psi_500" method in TropD_ Metric_PSI.

EDJ is the latitude of maximum zonal wind at the $850-\mathrm{hPa}$ level. In the SH it is calculated using the "peak" method (weak smoothing) in TropD_Metric_EDJ, whereas in the NH it is calculated using the "max" method (moderate smoothing). This method does not work well where the maximum is very sharp, which is the case for the $\mathrm{SH}$.

USF is the zero-crossing latitude of the surface zonal wind (and corresponds to UAS in TropD). It is calculated using the "zero_crossing" method in TropD_Metric_UAS.
STJ is the latitude of maximum of the zonal wind averaged between the 100 and $400 \mathrm{hPa}$ levels minus the zonal wind at the $850-\mathrm{hPa}$ level. It is calculated using the "adjusted_max" method in TropD_Metric_ STJ.

$\mathrm{P}-\mathrm{E}$ is the zero-crossing latitude of P-E poleward of the subtropical minimum in P-E and equatorward of $70^{\circ}$. As for PSI, if an additional zero-crossing latitude that meets the same criteria is found within $20^{\circ}$ latitude poleward of the initial zero-crossing latitude, the metric is set to a missing value. It is calculated using the "zero crossing" method in TropD_Metric_PE.

SLP is the latitude of maximal sea level pressure (and corresponds to PSL in TropD). It is calculated using TropD_Metric_PSL with, as for EDJ, the "peak" method in the SH and the "max" method in the NH.

TPB is the latitude of maximal poleward gradient of the tropopause pressure, calculated using the "max gradient" method in TropD_Metric_TPB.

OLR is the first latitude poleward of the subtropical outgoing longwave radiation maximum at which OLR drops to $250 \mathrm{~W} \mathrm{~m}^{-2}$, and is calculated using the method "250W" in TropD_Metric_OLR.

$\triangle \mathrm{OLR}$ is the first latitude poleward of the subtropical outgoing longwave radiation maximum in each hemisphere at which OLR drops to $20 \mathrm{~W} \mathrm{~m}^{-2}$ below the OLR maximum. It is calculated using the method "20W" in TropD_Metric_OLR.

\section{REFERENCES}

Adam, O., K. M. Grise, P. Staten, I. R. Simpson, S. M. Davis, N. A. Davis, D. W. Waugh, and T. Birner, 2018: The TropD software package: Standardized methods for calculating tropical width diagnostics. Geosci. Model Develop. Discuss., https://doi.org/ 10.5194/gmd-2018-124.

Amaya, D. J., N. Siler, S.-P. Xie, and A. J. Miller, 2018: The interplay of internal and forced modes of Hadley Cell expansion: Lessons from the global warming hiatus. Climate Dyn., 51, 305-319, https://doi.org/10.1007/s00382-017-3921-5.

Birner, T., S. M. Davis, and D. J. Seidel, 2014: The changing width of Earth's tropical belt. Phys. Today, 67, 38-44, https://doi.org/ 10.1063/PT.3.2620.

Choi, J., S.-W. Son, J. Lu, and S.-K. Min, 2014: Further observational evidence of Hadley cell widening in the Southern Hemisphere. Geophys. Res. Lett., 41, 2590-2597, https://doi.org/ 10.1002/2014GL059426.

Davis, N., and T. Birner, 2017: On the discrepancies in tropical belt expansion between reanalyses and climate models and among tropical belt width metrics. J. Climate, 30, 1211-1231, https:// doi.org/10.1175/JCLI-D-16-0371.1.

— D. J. Seidel, T. Birner, S. M. Davis, and S. Tilmes, 2016: Changes in the width of the tropical belt due to simple radiative forcing changes in the GeoMIP simulations. Atmos. Chem. Phys., 16, $10083-10095$, https://doi.org/10.5194/acp-16-10083-2016.

Davis, S. M., and K. H. Rosenlof, 2012: A multi-diagnostic intercomparison of tropical width time series using reanalysis 
and satellite observations. J. Climate, 25, 1061-1078, https:// doi.org/10.1175/JCLI-D-11-00127.1.

Donohoe, A., K. C. Armour, A. G. Pendergrass, and D. S. Battisti, 2014: Shortwave and longwave radiative contributions to global warming under increasing $\mathrm{CO}_{2}$. Proc. Natl. Acad. Sci. USA, 111, 16700-16 705, https://doi.org/10.1073/pnas.1412190111.

Grise, K. M., and L. M. Polvani, 2014: Is climate sensitivity related to dynamical sensitivity? A Southern Hemisphere perspective. Geophys. Res. Lett., 41, 534-540, https://doi.org/10.1002/2013GL058466. , and - 2016: Is climate sensitivity related to dynamical sensitivity? J. Geophys. Res. Atmos., 121, 5159-5176, https:// doi.org/10.1002/2015JD024687.

Heffernan, O., 2016: The mystery of the expanding tropics. Nature, 530, 20-22, https://doi.org/10.1038/530020a.

$\mathrm{Hu}$, Y., C. Zhou, and J. Liu, 2011: Observational evidence for the poleward expansion of the Hadley circulation. Adv. Atmos. Sci., 28, 33-44, https://doi.org/10.1007/s00376-010-0032-1.

Kang, S. M., and L. Polvani, 2011: The interannual relationship between the latitude of the eddy-driven jet and the edge of the Hadley cell J. Climate, 24, 563-568, https://doi.org/10.1175/2010JCLI4077.1.

Kidston, J., C. W. Cairns, and P. Paga, 2013: Variability in the width of the tropics and the annular modes. Geophys. Res. Lett., $\mathbf{4 0}$, 2328-2332, https://doi.org/10.1029/2012GL054165.

Lucas, C., B. Timbal, and H. Nguyen, 2014: The expanding tropics: A critical assessment of the observational and modeling studies. Wiley Interdiscip. Rev.: Climate Change, 5, 89-112, https://doi.org/10.1002/wcc. 251 .

Mantsis, D. F., S. Sherwood, R. Allen, and L. Shi, 2017: Natural variations of tropical width and recent trends. Geophys. Res. Lett., 44, 3825-3832, https://doi.org/10.1002/2016GL072097.

Polvani, L. M., D. W. Waugh, G. J. P. Correa, and S.-W. Son, 2011: Stratospheric ozone depletion: The main driver of twentieth-century atmospheric circulation changes in the Southern Hemisphere. J. Climate, 24, 795-812, https://doi.org/ 10.1175/2010JCLI3772.1.

Schmidt, D. F., and K. M. Grise, 2017: The response of local precipitation and sea level pressure to Hadley cell expansion. Geophys. Res. Lett., 44, 10 573-10 582, https://doi.org/10.1002/2017GL075380.

Seidel, D., Q. Fu, W. J. Randel, and T. J. Reichler, 2008: Widening of the tropical belt in a changing climate. Nat. Geosci., 1, 2124, https://doi.org/10.1038/ngeo.2007.38.

Seviour, W. J. M., S. M. Davis, K. M. Grise, and D. W. Waugh, 2018: Large uncertainty in the relative rates of dynamical and hydrological tropical expansion. Geophys. Res. Lett., 45, 11061113, https://doi.org/10.1002/2017GL076335.

Solomon, A., L. M. Polvani, D. W. Waugh, and S. M. Davis, 2016: Contrasting upper and lower atmospheric metrics of tropical expansion in the Southern Hemisphere. Geophys. Res. Lett., 43, 10 496-10 503, https://doi.org/10.1002/2016GL070917.

Son, S.-W., S.-Y. Kim, and S.-K. Min, 2018: Widening of the Hadley cell from Last Glacial Maximum (LGM) to future climate. J. Climate, 31, 267-281, https://doi.org/10.1175/JCLI-D-17-0328.1.

Staten, P. W., and T. Reichler, 2014: On the ratio between shifts in the eddy-driven jet and the Hadley cell edge. Climate Dyn., 42, 1229-1242, https://doi.org/10.1007/s00382-013-1905-7.

Taylor, K. E., R. J. Stouffer, and G. A. Meehl, 2012: An overview of CMIP5 and the experiment design. Bull. Amer. Meteor. Soc., 93, 485-498, https://doi.org/10.1175/BAMS-D-11-00094.1.

Vial, J., J.-L. Dufresne, and S. Bony, 2013: On the interpretation of intermodel spread in CMIP5 climate sensitivity estimates. Climate Dyn., 41, 3339-3362, https://doi.org/10.1007/s00382-013-1725-9.

Voigt, A., and T. A. Shaw, 2015: Circulation response to warming shaped by radiative changes of clouds and water vapor. Nat. Geosci., 8, 102-106, https://doi.org/10.1038/ngeo2345. 